\title{
Social media research: A scientometric assessment of world publications output during 2001-2014
}

\author{
B. M. Gupta*, S. M. Dhawan, Ritu Gupta² \\ National Institute of Science, Technology and Development Studies, ${ }^{1}$ National Physical Laboratory, New Delhi, \\ ${ }^{2}$ Sri Venkateswara University, Tirupati, Andhra Pradesh, India
}

\begin{abstract}
The paper examines world publications output on social media research (46354) published during 2001-2014. The publications data, sourced from Scopus database, averaged annual $14.61 \%$ growth. The paper profiles top 25 most productive organizations that accounted for $12.46 \%$ world share, and top 25 most productive authors that accounted for $2.34 \%$ world share on indicators such as average productivity, citations per paper, h-index, and share of international collaborative publications during 2001-2014. The distribution of world output by country of publication is highly skewed. The top 15 most productive countries account for bulk of the world output (82.40\%). The USA has emerged as the world leader both in its world share and citations impact of social media research output. Blogs, among social media sites, accounted for the largest publication share $27.45 \%$, followed by Facebook (16.75\%), Twitter (15.86\%), Wikipedia (10.58\%), YouTube (7.24\%), Flickr (3.94\%), MySpace (1.73\%), Linkedln (1.21\%), etc., during 2001-2014. Computer science accounted for the highest publications share $(55.22 \%)$ of world publications output on social media, followed by social sciences $(26.55 \%)$, engineering (13.52\%), medicine (10.14\%), business, management and accounting $(8.72 \%)$, arts and humanities (5.95\%), psychology (3.68\%), etc., during 2001-2014. The top 30 most productive journals, which reported social media research during 2001-2014, accounted for $6.46 \%$ world share. A total of 266 were discovered as highly cited papers in social media research $(0.57 \%$ world share), each was cited 100 or more times since publication till February 2015. Together these highly cited papers accounted 57462 citations, with an average of 216 citations per paper.
\end{abstract}

Keywords: Bibliometrics, publications, scientometrics, social media, social media networks, social media sites

\section{INTRODUCTION}

Social media has invaded our lives, empowered people with new pervasive communication tools capable of creating big social impact. Little by little social media has been widening facilities for communication with the rest of the world. Within a decade, social media has become one of the most powerful media sources for news updates, online collaboration, networking, viral

*Address for correspondence:

E-mail: bmgupta1@gmail.com

\begin{tabular}{|l|l|}
\hline \multicolumn{2}{|c|}{ Access this article online } \\
\hline Quick Response Code: & Website: \\
\hline & www.jscires.org \\
& Dol: \\
& $10.4103 / 2320-0057.174865$ \\
\hline
\end{tabular}

marketing, and entertainment. It is increasingly difficult to ignore its potential in our day to day life. For many organizations, social media has become a primary channel to engage, listen, and communicate with a variety of stakeholders from customers to employees to suppliers and competitors. The contemporary interest in social media within the academic, public, and business circles has been driven largely by the rise of social media platforms, such as Twitter, Facebook, and YouTube. Social media' stance toward distributing data is relatively open, and it is this

This is an open access article distributed under the terms of the Creative Commons Attribution-NonCommercial-ShareAlike 3.0 License, which allows others to remix, tweak, and build upon the work non-commercially, as long as the author is credited and the new creations are licensed under the identical terms.

For reprints contact: reprints@medknow.com

How to cite this article: Gupta BM, Dhawan SM, Gupta R. Social media research: A scientometric assessment of world publications output during 2001-2014. J Sci Res 2015;4:161-71. 
stance that is driving its penetration with astonishingly fast rate on the web.

Individuals produce data at an unprecedented rate by interacting, sharing, and consuming content through social media. Social media collects the data in a structured and unstructured way, which contains sentiments and opinion of users and can be effectively processed using data mining techniques for achieving the meaningful results. Using social media data, we can classify the type of users and analysis of their posted data on the social websites. Machine learning algorithms are good at text classification which can be used to extract meaningful data from these websites.

Currently, scholars from a number of disciplines of sociology, communication, anthropology, media studies, library and information science, and cultural studies are using social media data and techniques as a part of their research practices. Social media is slowly evolving as a discipline of study with its own thematic areas of study, a community of scholars, courses in the universities, and within its unique publishing channels, etc.

Social media continues to grow apace around the world too, with active user accounts now equating to roughly $29 \%$ of the world's population. Monthly active user figures as on January 2015, for the most active social network in each country, add up to almost 2.08 billion - a $12 \%$ increase since January 2014. The mobile usage of social networks continues to grow all over the world, with at least 1.65 billion active mobile social accounts in January 2015. Facebook continues to dominate the global social media landscape, claiming 1.366 billion active users in January 2015, followed by Facebook Messenger ( 0.500 billion), LinkedIn (0.347 billion). Google Plus (0.343 billion), Instagram ( 0.300 billion), Twitter (0.284 billion), etc. ${ }^{[1]}$

Social media are interactive platforms where content is created, distributed, and shared by individuals on the web. Social media according to Kaplan and Haenlein is defined as "a group of internet-based applications that build on the ideological and technological foundations of Web 2.0 for the creation and exchange user-generated contents." Social media websites and applications allow users to create and exchange user-oriented content whereby people talk, share information, participate, and network through technologies such as blogs and social networking sites. ${ }^{[2]}$

Before the term Web 2.0 was created in 1999, internet pages featured mostly static content such as text and graphics and websites operated on Web 1.0 technologies; website hosts and owners were the primary content creators. Online information targeted a mostly passive audience that received rather than contributed content. However, with the introduction of Web 2.0 internet technologies around the turn of $21^{\text {st }}$ century, social media venues such as blogs began to allow users to interact and collaborate with each other in virtual communities. This more open, shared method of social media dialog contrasted significantly with the top-down approach that characterized the earlier years of web. Specifically, social media began meeting the characteristics of Web 2.0 websites, providing a rich user experience, dynamic content, scalability, openness, and collective intelligence. Nevertheless, social media has grown rapidly in the U.S and around the world due to its blending of technology and social interaction for the creation of value. ${ }^{[2]}$

The first recognizable social media site, SixDegrees was created in 1997. It enabled users to upload a profile and make friends with other users. In 1999, the first blogging site became popular creating a social media sensation that is still popular today. After the invention of blogging, social media began to explode in popularity. Sites such as MySpace and LinkedIn gained prominence in the early 2000s, and sites such as Photobucket and Flickr facilitated online photo sharing. YouTube came out in 2005, creating an entirely new way for people to communicate and share with each other across great distances. By 2006, Facebook and Twitter both became available to users throughout the world. These sites remain some of the most popular social networks on the internet. Other sites such as Tumblr, Spotify, Foursquare, and Pinterest began popping up to fill specific social networking niches. ${ }^{[3]}$

Social media can take many different forms including internet forums, weblogs, social blogs, wikis, podcasts, pictures, videos, rating, and bookmarking. Technologies include blogs, picture-sharing, vlogs, wall postings, E-mail, instant messaging, music sharing, crowdsourcing, and voice over IP, to name a few. Many of the social media services can be integrated via social network aggregation platforms such as MyblogLog and Plaxo. Examples of social media applications, social media sites include: (a) Communication- (i) Blogs-bloggers, Live Journal, and Open Dairy (ii) Micro-blogging/Pressure Applications - Twitter and Plurk (iii) Social Networking - Facebook, LinkedIn, MySpace, and Orkut (iv) Social Networking Aggregation - Nutshell Mail and (v) Events - Upcoming 
and Eventful; (b) Collaboration - (i) Wikis - Wikipedia (ii) Social Bookmarking or Social Tagging - Delicious Good Reader (iii) Social News - Digg and Reddit and (iv) Opinion Sites - Epinions and Yelp; (c) Multimedia - (i) Photosharing - Flickr and Zoomr (ii) Video sharing - YouTube and Vimeo (iii) Livecasting - Upstream Tv and (iv) Audio and Music Sharing - Imeem and The Hype Machine; (d) Reviews and Opinions - Product Reviews - epinions.com and Q and A - Wiki Answers; and (e) Entertainment - (i) Media and Entertainment Platforms - Cisco Ees (ii) Virtual Worlds - Second Life and (iii) Game Sharing - Miniclip. ${ }^{[4]}$

\section{Literature Review}

So far, only a few studies have undertaken a quantitative assessment of world literature on social media research. Coursaris and Van $\mathrm{Osch}^{[5]}$ examined the research productivity and citation impact of authors, institutions, and countries based on 610 peer-reviewed social media articles published between October 2004 and December 2011. Results indicate that research productivity is exploding and that several leading authors, institutions, countries, and a small set of foundational papers have since emerged. Social media as a domain displays limited diversity and is still heavily influenced by practitioners. The paper raises two fundamental challenges facing the social media domain and its future advancement, namely the lack of academic maturity and the Matthew effect. Gan and Wang $^{[6]}$ examine 646 journal publications on social media research under the subject category "Information Science and Library Science" and as indexed in Social Science Citation Index. The study analyzed research performance and trends by languages, characteristics, countries, journals, authorships, and author keywords. Keeping these studies in mind, the authors decided to undertake comparatively a more comprehensive study of world social media literature covering 14 years period from 2001 to 2014 .

\section{OBJECTIVES}

The study undertakes comprehensive assessment of world social media research covering the period 2001-2014 to address the following objectives:

- To study the growth of world literature on social media research, the distribution of research output by top 15 most productive countries, and compare their performance on citation impact

- To study the distribution of world research output by broad subject areas, and analyze research activity on activity index indicator
- To study and compare publication productivity and citation impact of top organizations and authors

- To study the medium of communication and the characteristics of highly cited publications.

\section{METHODOLOGY}

The study sourced publications data on social media research, covering the period 2001-2014, from the Scopus database (http://www.scopus.com). For this purpose, a search string was formulated using a number of significant keywords (as shown in the search string given below) in "title, abstract, and keyword" tag and restricting search period to 2001-2014 in "date range tag." The search string was further restricted to 15 top most productive countries in "country tag" to collect their publication data stats country by country. Further, the search string was restricted to "subject area tag," "country tag," "source title tag," "journal title name," and "affiliation tag" to get publications stats by subject, collaborating countries, organization, author-wise and journal-wise, etc. For citation data, citation window was limited to three publication years (publications during 2004-2012), to two publication years (publications of 2013) and to one publication year (publications of 2014). In addition, citations data were collected for total publication output during 2001-2014 from date of publications till the end February 2015.

(((TITLE-ABS-KEY [ "social media”] OR TITLE-ABS-KEY ("social medium" or "social network site*”) OR TITLE-ABS-KEY [“social networking site*” or “online social network"]) AND PUBYEAR > 2000 AND PUBYEAR < 2015) or ((TITLE-ABS-KEY [facebook] OR TITLE-ABS-KEY (Twitter or Wikipedia or foursquare) OR TITLE-ABS-KEY [inkedin or myspace or "google plus"] OR TITLE-ABS-KEY (instagram or pinterest or FLICKR) OR TITLE-ABS-KEY ["academia.edu” or "researchgate"] OR TITLE-ABS-KEY ("you tube” or "youtube") OR TITLE-ABS-KEY [slideshare]) AND PUBYEAR > 2000 AND PUBYEAR < 2015) or ((TITLE-ABS-KEY ["blog" or "blogs"] OR TITLE-ABS-KEY ("blogging" or "bloggers") OR TITLE-ABS-KEY ["weblog" or “microblog”]) AND PUBYEAR > 2000 AND PUBYEAR <2015)).

\section{ANALYSIS}

The annual world publications output in social media research expanded from 3 in 2001 to 2223 in 2008 and to 9105 publications in 2014, logging average $14.61 \%$ 
annual growth. The cumulative septennial world output expanded from 2595 during 2001-2007 to 43759 during 2008-2014, logging 1586.28\% septennial growth [Table 1]. Of the total world publication output (46354), 44.76\% appeared as conference papers, $38.83 \%$ as articles, $3.49 \%$ as reviews, $2.74 \%$ as book chapters, $2.55 \%$ as conference reviews, and $1.86 \%$ as notes and the rest as articles in press, editorials, short surveys, books, letters, and erratum during 2001-2014.

\section{Citation Impact of Top 15 Most Productive Countries}

A total of 142 countries contributed to social media research during 2001-2014. The distribution of publications output by publication rate revealed that in 14 years 103 countries contributed 1-100 papers each, 28 countries from 101 to 1000 papers each, 7 countries from 1001 to 2000 papers each, 1 country from 2001 to 3000 papers, 2 countries from 3001 to 4000 papers each, and 1 country 15001-16000 papers during 2001-2014. Among the top 15 most productive countries, the United States contributed the largest publication share $(32.40 \%)$, followed far behind by China (8.09\%); the United Kingdom (7.67\%); Germany (4.94\%); Australia (4.30\%); Canada, Spain, and Japan (from 3.04\% to 3.55\%); Italy, Taiwan, France, India, The Netherlands, and South Korea (from $2.06 \%$ to $2.58 \%$ ); and Singapore $(1.70 \%)$. The top 15 most productive countries together contributed $82.40 \%$ share to the world output on social media during 2001-2014. The citation impact registered by the United States was the largest (7.44), followed by the United Kingdom (5.66), The Netherlands (5.59), Canada (5.46), Germany (5.31), France (5.16), Singapore (4.51), South Korea (4.46\%), Italy (4.05), Spain

\begin{tabular}{lc}
$\begin{array}{l}\text { Table 1: Annual publications output in social media } \\
\text { research, 2004-2014 }\end{array}$ \\
\hline Year & Number of publications \\
\hline 2001 & 3 \\
2002 & 19 \\
2003 & 41 \\
2004 & 95 \\
2005 & 309 \\
2006 & 696 \\
2007 & 1432 \\
2008 & 2223 \\
2009 & 3142 \\
2010 & 4661 \\
2011 & 6293 \\
2012 & 8294 \\
2013 & 10,041 \\
2014 & 9105 \\
Total & 46,354 \\
\hline
\end{tabular}

(3.94), Australia (3.81), Taiwan (3.51), China (2.60), Japan (2.57), and India (1.85) during 2001-2014 [Table 2].

\section{Subject-wise Distribution of Research Output}

The world output on social media research covering the period 2001-2014 was distributed by ten subject sub-fields (as reflected in Scopus database classification). The subject-wise distribution revealed that computer science accounted for the largest share (55.22\%) followed by social sciences $(26.55 \%)$, engineering $(13.52 \%)$, medicine $(10.14 \%)$, business, management, and accounting (8.72\%), arts and humanities (5.95\%), psychology (3.68\%), etc., The septennial research activity, as reflected in activity index, went up in six disciplines, i.e. computer science, social sciences, medicine, arts and humanities, psychology, and decision sciences, - but in contrast, it went down in four disciplines, i.e., engineering, business, management and accounting, economics, econometrics, and finance during septennial periods 2001-2007 to 2008-2014 [Table 3].

\section{Distribution of Publications Output by Social Media Site}

Blogs accounted for the largest share $(27.45 \%)$ in the total world publications output on social media research, followed by Facebook (16.75\%), Twitter (15.86\%), Wikipedia (10.58\%), YouTube (7.24\%), Flickr (3.94\%), MySpace (1.73\%), LinkedIn (1.21\%), and others $<1 \%$ during 2001-2014. Comparatively, citation impact by MySpace literature was the largest (14.14 citations per paper) followed by Orkut (13.80), Flickr (6.28), Wikipedia (5.77), Facebook (5.33), YouTube (4.79), Blogs (4.55), LinkedIn (3.59), Foursquare (3.00), Twitter (1.79), ResearchGate (1.30), Academia.Edu (1.20), Slideshare (1.00), Pinterest (0.80), and Instagram (0.54) during 2001-2014 [Table 4 and Figure 1]. The annual publications output distributed by social media sites and by country of publication of research content are shown in Tables 5 and 6.

\section{Most Significant Keywords}

In terms of significant keywords, online social network was seen as the most significant keyword in terms of number of publications it retrieved (10395), followed by social media (9605), social network (6076), internet (6352), Facebook (3495), Wikipedia (2973), blogs (2535), world wide web (2524), Twitter (2041), data mining (1997), information retrieval (1956), websites (1922), Web 2.0 (1684), information system (1520), YouTube (1279), blogging 
Table 2: Citation profile of top 15 most productive countries in social media research, 2001-2014

\begin{tabular}{|c|c|c|c|c|c|c|c|}
\hline \multirow{2}{*}{$\begin{array}{l}\text { Name of the } \\
\text { country }\end{array}$} & \multicolumn{4}{|c|}{ Number of publications } & \multirow{2}{*}{$\begin{array}{c}\text { TC } \\
2001-2014\end{array}$} & \multirow{2}{*}{$\begin{array}{c}\text { ACPP } \\
2001-2014\end{array}$} & \multirow{2}{*}{$\begin{array}{c}\text { Share of world papers } \\
2001-2014\end{array}$} \\
\hline & 2001-2004 & 2005-2009 & $2010-2014$ & 2001-2014 & & & \\
\hline USA & 55 & 2479 & 12,481 & 15,025 & 111,775 & 7.44 & 32.4 \\
\hline China & 3 & 389 & 3358 & 3750 & 9757 & 2.60 & 8.09 \\
\hline The United Kingdom & 8 & 557 & 2990 & 3555 & 20,105 & 5.66 & 7.67 \\
\hline Germany & 3 & 402 & 1883 & 2288 & 12,142 & 5.31 & 4.94 \\
\hline Australia & 1 & 296 & 1697 & 1994 & 7595 & 3.81 & 4.30 \\
\hline Canada & 8 & 222 & 1416 & 1646 & 8988 & 5.46 & 3.55 \\
\hline Spain & 0 & 176 & 1281 & 1457 & 5747 & 3.94 & 3.14 \\
\hline Japan & 6 & 312 & 1091 & 1409 & 3617 & 2.57 & 3.04 \\
\hline Italy & 2 & 177 & 1019 & 1198 & 4847 & 4.05 & 2.58 \\
\hline Taiwan & 2 & 179 & 901 & 1082 & 3800 & 3.51 & 2.33 \\
\hline France & 3 & 164 & 908 & 1075 & 5547 & 5.16 & 2.32 \\
\hline India & 1 & 92 & 899 & 992 & 1833 & 1.85 & 2.14 \\
\hline The Netherlands & 1 & 72 & 816 & 989 & 5527 & 5.59 & 2.13 \\
\hline South Korea & 0 & 132 & 823 & 955 & 4257 & 4.46 & 2.06 \\
\hline Singapore & 0 & 121 & 665 & 786 & 3542 & 4.51 & 1.70 \\
\hline World total & 158 & 7802 & 38,394 & 46,353 & & & \\
\hline
\end{tabular}

$\mathrm{TC}=$ Total citations, $\mathrm{ACPP}=$ Average citation per paper

Table 3: Subject-wise break-up of world's publications in social media research, 2001-2014

\begin{tabular}{|c|c|c|c|c|c|c|}
\hline \multirow[t]{2}{*}{ Subject } & \multicolumn{3}{|c|}{ Number of papers } & \multicolumn{2}{|c|}{ Activity index } & \multirow{2}{*}{$\begin{array}{l}\text { Percentage of share of word output } \\
\qquad 2001-2014\end{array}$} \\
\hline & $2001-2007$ & 2008-2014 & $2001-2014$ & 2001-2007 & 2008-2014 & \\
\hline Computer science & 1326 & 24,271 & 25,597 & 92.53 & 100.4 & 55.22 \\
\hline Social sciences & 649 & 11,659 & 12,308 & 94.19 & 100.3 & 26.55 \\
\hline Engineering & 492 & 5773 & 6265 & 140.3 & 97.61 & 13.52 \\
\hline Medicine & 107 & 4593 & 4700 & 40.67 & 103.5 & 10.14 \\
\hline Business, management, and accounting & 234 & 3808 & 4042 & 103.4 & 99.8 & 8.72 \\
\hline Arts and humanities & 57 & 2702 & 2759 & 36.9 & 103.7 & 5.95 \\
\hline Psychology & 26 & 1681 & 1707 & 27.21 & 104.3 & 3.68 \\
\hline Decision sciences & 73 & 1577 & 1650 & 79.03 & 101.2 & 3.56 \\
\hline Biochemistry, genetics, and molecular biology & 200 & 644 & 844 & 423.3 & 80.83 & 1.82 \\
\hline Economics, econometrics, and finance & 88 & 756 & 844 & 186.2 & 94.89 & 1.82 \\
\hline Total of the world & 2595 & 43,759 & 46,354 & & & \\
\hline
\end{tabular}

Table 4: Distribution of publications on different social media sites during 2001-2014

\begin{tabular}{lcccc}
\hline Type of social media site & TP & TC & ACPP & TP (\%) \\
\hline Blogs & 12,726 & 57,853 & 4.55 & 27.45 \\
Facebook & 7765 & 41,424 & 5.33 & 16.75 \\
Twitter & 7352 & 13,135 & 1.79 & 15.86 \\
Wikipedia & 4906 & 28,315 & 5.77 & 10.58 \\
YouTube & 3354 & 16,062 & 4.79 & 7.24 \\
Flickr & 1828 & 11,482 & 6.28 & 3.94 \\
MySpace & 801 & 11,328 & 14.14 & 1.73 \\
Linkedln & 559 & 2007 & 3.59 & 1.21 \\
Fouresquare & 408 & 1226 & 3.00 & 0.88 \\
Orkut & 109 & 1504 & 13.8 & 0.235 \\
Instagram & 105 & 57 & 0.54 & 0.227 \\
Pinterest & 97 & 78 & 0.80 & 0.209 \\
ResearchGate & 20 & 26 & 1.30 & 0.043 \\
Academia.Edu & 10 & 12 & 1.20 & 0.022 \\
Slideshare & 1 & 1 & 1.00 & 0.002 \\
World Total & 46,354 & & & \\
\hline TP & &
\end{tabular}

$\mathrm{TP}=$ Total papers, $\mathrm{TC}=$ Total citations, $\mathrm{ACPP}=$ Average citation per paper
(1208), online systems (1249), knowledge management (1181), artificial intelligence (1101), search engines (1077), natural language processing system (1059), information technology (1011), micro-blog (1011), e-learning (714), information dissemination (901), human-computer interactions (644), information management (637), bloggers (339), Flickr (246), weblog (175), blogosphere (156), LinkedIn (88), MySpace (53), Foursquare (46), Instagram (24), Pinterest (23), Orkut (11), etc.

\section{Scientometric Profile of Top 25 Organizations}

The top 25 most productive organizations in social media research together contributed 5776 publications, and they accounted for $12.46 \%$ share of the world output published during 2001-2014. The top 10 organizations contributed productivity above the average of (231.04 papers) per organization: Carnegie Mellon University, USA 
(373 papers), Tsinghua University, China (322 papers), National University of Singapore (308 papers), University of Maryland, USA (280 papers), Pennsylvania State University, USA and Arizona State University, USA

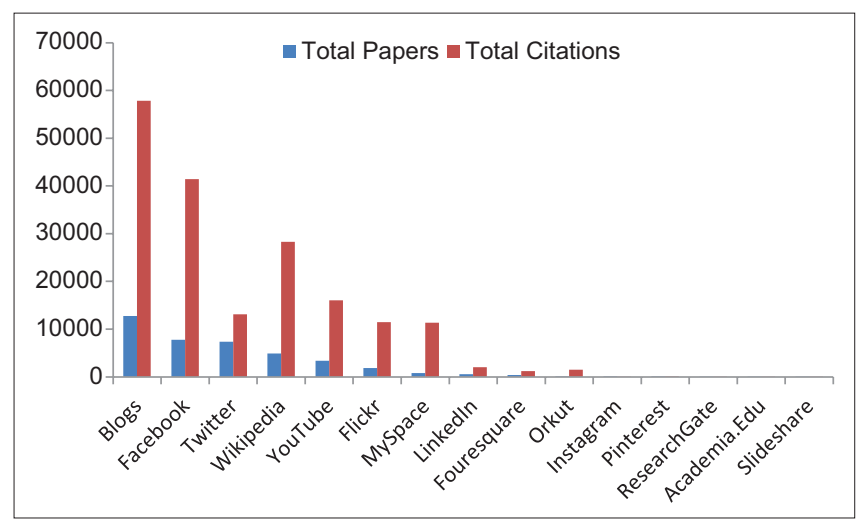

Figure 1: Distribution of Publications by Social Media Sites, 2001-2014
(278 papers each), Microsoft Research, USA (266 papers), Indiana University, USA (264 papers), University of Texas at Austin, USA (240 papers), and University of Amsterdam, The Netherlands (237 papers).

Eight organizations registered citation impact above the average of 92.4 citations per paper: Michigan State University, USA (9.24), University of California, Berkley, USA (28.66), Cornell University, USA (21.37), Stanford University, USA (13.80), University of Texas at Austin, USA (11.62), University of Maryland, USA (11.25), University of California, Irvine, USA (10.56), and University of Illinois at Urbana - Champaign, USA (9.49).

Ten organizations scored $\mathrm{h}$-index above the average score of 21: Carnegie Mellon University, USA (31), University of Maryland, USA (29), Cornell University, USA (28), University of California, Berkley, USA and Stanford

Table 5: Annual growth of world publications by leading social media sites, 2001-2014

\begin{tabular}{|c|c|c|c|c|c|c|c|c|c|c|c|}
\hline \multirow{2}{*}{$\begin{array}{l}\text { Social media } \\
\text { site name }\end{array}$} & \multicolumn{11}{|c|}{ Number of publications } \\
\hline & 2005-2014 & 2005 & 2006 & 2007 & 2008 & 2009 & 2010 & 2011 & 2012 & 2013 & 2014 \\
\hline Blogs & 12,726 & 246 & 502 & 842 & 1095 & 1235 & 1631 & 1674 & 1812 & 1987 & 1576 \\
\hline Facebook & 7765 & 2 & 11 & 56 & 204 & 388 & 669 & 1131 & 1614 & 1922 & 1768 \\
\hline Twitter & 7352 & 3 & 3 & 10 & 35 & 189 & 599 & 1039 & 1547 & 2067 & 1852 \\
\hline Wikipedia & 4906 & 30 & 113 & 280 & 451 & 582 & 692 & 706 & 725 & 732 & 589 \\
\hline Foursquare & 408 & 4 & 4 & 4 & 2 & 9 & 23 & 56 & 95 & 117 & 89 \\
\hline Linkedln & 559 & 1 & 1 & 5 & 13 & 35 & 52 & 64 & 118 & 137 & 132 \\
\hline YouTube & 3354 & 1 & 11 & 103 & 186 & 293 & 396 & 510 & 576 & 696 & 582 \\
\hline ResearchGate & 20 & & & & & & 1 & 1 & 6 & 12 & \\
\hline Academia.Edu & 10 & & & & & & & 1 & 1 & 3 & 5 \\
\hline Orkut & & 3 & 4 & 5 & 6 & 15 & 20 & 18 & 14 & 13 & 11 \\
\hline Flickr & 1828 & 1 & 24 & 88 & 136 & 227 & 276 & 277 & 298 & 277 & 223 \\
\hline MySpace & 801 & 4 & 29 & 74 & 116 & 141 & 150 & 114 & 82 & 58 & 31 \\
\hline Instagram & 105 & & & & & & 2 & 1 & 8 & 27 & 67 \\
\hline Pinterest & 97 & & & & & & & 1 & 17 & 40 & 39 \\
\hline Slideshare & 24 & & & 1 & 2 & 3 & 3 & 2 & 2 & 5 & 6 \\
\hline
\end{tabular}

Table 6: Contribution of leading countries by seven major social media sites, 2001-2014

\begin{tabular}{|c|c|c|c|c|c|c|c|}
\hline \multirow[t]{2}{*}{ Name of the country } & \multicolumn{7}{|c|}{ Number of publications } \\
\hline & Blogs & Facebook & Twitter & Wikipedia & YouTube & Flickr & MySpace \\
\hline USA & 3553 & 2819 & 2544 & 1249 & 1204 & 500 & 364 \\
\hline China & 1474 & 241 & 484 & 378 & 243 & 304 & 21 \\
\hline The United Kingdom & 904 & 548 & 548 & 265 & 269 & 154 & 74 \\
\hline Germany & 383 & 327 & 327 & 506 & 162 & 169 & 21 \\
\hline Australia & 516 & 364 & 282 & 146 & 171 & 55 & 45 \\
\hline Canada & 413 & 271 & 247 & 167 & 167 & 44 & 30 \\
\hline Spain & 360 & 213 & 290 & 341 & 90 & 87 & 4 \\
\hline Japan & 512 & 99 & 397 & 236 & 67 & 68 & 9 \\
\hline Italy & 252 & 209 & 177 & 233 & 91 & 75 & 12 \\
\hline Taiwan & 489 & 253 & 82 & 76 & 45 & 30 & 9 \\
\hline France & 227 & 150 & 155 & 227 & 95 & 98 & 13 \\
\hline India & 307 & 180 & 222 & 133 & 85 & 36 & 16 \\
\hline World total & 12,726 & 7765 & 7352 & 4906 & 3354 & 1828 & 801 \\
\hline
\end{tabular}


University, USA (27 each), University of Texas at Austin, USA and University of Illinois at Urbana - Champaign, USA (25), Pennsylvania State University, USA and Microsoft Research, USA (24 each), and University of Washington, Seattle, USA (23).

Nine organizations contributed international collaborative share of papers above the average of $(26.14 \%)$ : National University of Singapore (53.57\%), Tsinghua University, China (43.17\%), Peking University, China (41.15\%), IBM J Watson Research Center, USA (36.67\%), Nanyang Technological University, Singapore (34.65\%), University of Toronto, Canada (34.53\%), City University of Hong Kong $(30.16 \%)$, University of California, Irvine, USA (28.65\%), and University of Amsterdam, The Netherlands (27.85\%) during 2001-2014 [Table 7].

\section{Scientometric Profile of Top 25 Authors}

The top 25 most productive authors together contributed 1084 publications in social media research, and they accounted for $2.34 \%$ share of the world output during
2001-2014. Of the total authors, nine contributed above the average productivity of 43.36 per organization: M. De Rijke (73 papers), H. Liu (68 papers), M. Thelwall (63 papers), E. P. Lim (49 papers), G. Weikum (47 papers), A. Sun and K. Lerman (46 papers each), F. Abel (45 papers), and W. Nejdl (44 papers) during 2001-2014.

Seven authors registered citation impact above the average 13.80 citations per paper: C. Lampe (79.50), J. Leskovec (44.40), M. Naaman (34.60), G. Weikum (24.80), F. Benevenuto (18.10), M. Thelwall (17.20), and C. Faloutsos (14.60).

Thirteen authors posted h-index above the average of 9.92 score: M. Thelwall (19), J. Leskovec (15), G. Weikum (14), F. Benevenuto (13), M. Naaman and J. Han (12 each), H. Liu and M. De Rijke (11 each), E. P. Lim, H. Sundaram, J. Tang, and A. Sun (10 each).

Eleven authors contributed international collaborative papers above the average share of 29.34\%: J. Weber $(83.33 \%)$, T. S. Chua (69.77\%), J. Tang (66.67\%), F. Abel

Table 7: Scientometric profile of top 25 most productive organizations in social media, 2001-2014

\begin{tabular}{|c|c|c|c|c|c|c|}
\hline Name of the organizations & TP & TC & ACPP & $\mathrm{HI}$ & ICP & ICP (\%) \\
\hline Carnegie Mellon University, USA & 373 & 3357 & 9.00 & 31 & 96 & 25.74 \\
\hline Tsinghua University, China & 322 & 1205 & 3.74 & 20 & 139 & 43.17 \\
\hline National University of Singapore & 308 & 1009 & 3.28 & 17 & 165 & 53.57 \\
\hline University of Maryland, USA & 280 & 3149 & 11.25 & 29 & 38 & 13.57 \\
\hline Pennsylvania State University, USA & 278 & 2500 & 8.99 & 24 & 49 & 17.63 \\
\hline Arizona State University, USA & 278 & 1405 & 5.054 & 19 & 42 & 15.11 \\
\hline Microsoft Research, USA & 266 & 2207 & 8.297 & 24 & 51 & 19.17 \\
\hline Indiana University, USA & 264 & 1919 & 7.2689 & 21 & 68 & 25.76 \\
\hline University of Texas at Austin, USA & 240 & 2790 & 11.625 & 25 & 38 & 15.83 \\
\hline University of Amsterdam, The Netherlands & 237 & 1457 & 6.1477 & 21 & 66 & 27.85 \\
\hline Stanford University, USA & 223 & 3077 & 13.798 & 27 & 50 & 22.42 \\
\hline University of Toronto, Canada & 223 & 1534 & 6.8789 & 19 & 77 & 34.53 \\
\hline University of Illinois at Urbana-Champaign, USA & 214 & 2030 & 9.486 & 25 & 51 & 23.83 \\
\hline Nanyang Technological University, Singapore & 202 & 762 & 3.7723 & 16 & 70 & 34.65 \\
\hline Cornell University, USA & 202 & 4317 & 21.371 & 28 & 47 & 23.27 \\
\hline University of Tokyo, Japan & 197 & 1057 & 5.3655 & 12 & 26 & 13.2 \\
\hline Peking University, China & 192 & 580 & 3.0208 & 15 & 79 & 41.15 \\
\hline University of Washington, Seattle, USA & 191 & 1444 & 7.5602 & 23 & 37 & 19.37 \\
\hline City University of Hong Kong & 189 & 1184 & 6.2646 & 19 & 57 & 30.16 \\
\hline Beijing University of Posts \& Telecommunications & 187 & 232 & 1.2406 & 7 & 34 & 18.18 \\
\hline University of California, Berkley, USA & 185 & 5303 & 28.665 & 27 & 34 & 18.38 \\
\hline Michigan State University, USA & 184 & 6953 & 37.788 & 21 & 33 & 17.93 \\
\hline Queensland University of Technology, Australia & 183 & 758 & 4.1421 & 13 & 46 & 25.14 \\
\hline IBM J Watson Research Center, USA & 180 & 1277 & 7.0944 & 20 & 66 & 36.67 \\
\hline University of California, Irvine, USA & 178 & 1880 & 10.562 & 19 & 51 & 28.65 \\
\hline Total of 25 organizations & 5776 & 53,386 & 9.24 & 21 & 1510 & 26.14 \\
\hline Total of the world & 46,354 & & & & & \\
\hline Share of 25 organizations in world total & 12.46 & & & & & \\
\hline
\end{tabular}

$\mathrm{TP}=$ Total papers, $\mathrm{TC}=$ Total citations, $\mathrm{ACPP}=$ Average citation per paper, $\mathrm{HI}=\mathrm{H}$-index, $\mathrm{ICP}=$ International collaborative papers 
(62.22S\%), F. Benevenuto (48.72\%), J. Luo (47.37\%), C. Faloutsos (45.71\%), M. Thelwall (39.68\%), H. Chen (34.15\%), Y. Yu (29.34\%), and G. Weikum (29.79\%) during 2001-2014 [Table 8].

\section{Medium of Communication}

Among various media sources used to report social media research during 2001-2014, journals alone accounted for 21015 publications (45.34\% share). The top 30 most productive journals contributed from 47 to 375 papers each and together they contributed $14.25 \%$ share (2994 papers) of the total journal publications output on social media research during 2001-2014 [Table 9].

\section{Highly Cited Papers}

Of the total publications output (46354) on social media research covering the period 2001-2014, only $266(0.57 \%)$ were discovered as highly cited papers; each such paper was cited 100 or more times since their publication till February 2015. Among 266 highly cited papers,
187 were cited 100-200 times, 68 were cited from 201 to 500 citations, 6 papers were cited 501-1000 times, 4 were cited from 1001 to 2000 times, and 1 paper was cited above 2000 times. These 266 highly cited papers together accounted for 57462 citations, averaging 216.02 citations per paper. Of the 266 highly cited papers, 146 appeared as articles, 100 as conference papers, 14 as reviews, 4 as books, 1 as editorial, and 1 as a short survey. Among 266 high cited papers, 140 appeared as single-institution papers (zero collaboration), 89 as multi-institutional with national collaboration, and 37 as multi-institutional with international collaboration. Among the countries that contributed to 266 highly cited papers, the largest number (173) was from the United States, followed by the U.K. (23); Germany (21); Canada (14); The Netherlands (9); Spain (7); Australia and France (5 each); South Korea, Hong Kong, Switzerland, and Israel (4 each), Japan, Brazil, Austria and Ireland (3 each); Italy, Taiwan, Singapore, and Turkey (2 each); India, Finland, Greece, Sweden, Belgium, and South Africa (1 each), etc. Of the 162 highly cited papers that appeared in 84 journals, the largest

Table 8: Scientometric profile of top 25 most productive organizations in social media, 2001-2014

\begin{tabular}{|c|c|c|c|c|c|c|c|}
\hline Name of the author & Affiliation of the author & TP & TC & ACPP & $\mathrm{HI}$ & ICP & ICP (\%) \\
\hline M De Rijke & University of Amsterdam, The Netherlands & 73 & 366 & 5.01 & 11 & 12 & 16.44 \\
\hline H. Liu & Arizona State University, USA & 68 & 488 & 7.18 & 11 & 7 & 10.29 \\
\hline M. Thelwall & University of Wolverhampton, U.K. & 63 & 1086 & 17.2 & 19 & 25 & 39.68 \\
\hline E .P. Lim & Singapore Management University & 49 & 653 & 13.3 & 10 & 13 & 26.53 \\
\hline G. Weikum & Max Planck Institute for Computer Science, Saarbrucken, Germany & 47 & 1167 & 24.8 & 14 & 14 & 29.79 \\
\hline A. Sun & Nanyang Technological University, Singapore & 46 & 330 & 7.17 & 10 & 11 & 23.91 \\
\hline K. Lerman & University of Southern California, Columbia, SC, USA & 46 & 304 & 6.61 & 8 & 4 & 8.696 \\
\hline F. Abel & Delft University of Technology, The Netherlands & 45 & 190 & 4.22 & 7 & 28 & 62.22 \\
\hline W. Nejdl & University of Hannover, Germany & 44 & 192 & 4.36 & 8 & 6 & 13.64 \\
\hline T.S. Chua & National University of Singapore & 43 & 344 & 8 & 8 & 30 & 69.77 \\
\hline J. Han & University of Illinois at Urbana-Champaign, USA & 43 & 415 & 9.65 & 12 & 9 & 20.93 \\
\hline J. Weber & Yahoo Research Laboratory, Barcelona, Spain & 42 & 161 & 3.83 & 6 & 35 & 83.33 \\
\hline F. Benevenuto & Federal University of Minas Gerais, Brazil & 39 & 705 & 18.1 & 13 & 19 & 48.72 \\
\hline J. Leskovec & Carnegie Mellon University, USA & 38 & 1687 & 44.4 & 15 & 5 & 13.16 \\
\hline H. Chen & University of Arizona, USA & 41 & 215 & 5.24 & 7 & 14 & 34.15 \\
\hline J. Luo & Kodak Research Lab, USA & 38 & 398 & 10.5 & 9 & 18 & 47.37 \\
\hline M. De Choudhury & Arizona State University, USA & 37 & 262 & 7.08 & 9 & 4 & 10.81 \\
\hline J. Tang & Tsinghua University, China & 36 & 344 & 9.56 & 10 & 24 & 66.67 \\
\hline C Lampe & Michigan State University, USA & 37 & 2942 & 79.5 & 12 & 2 & 5.405 \\
\hline C.Faloutsos & Carnegie Mellon University, USA & 35 & 510 & 14.6 & 8 & 16 & 45.71 \\
\hline M. Naaman & Yahoo Research, Berkeley, USA & 35 & 1210 & 34.6 & 12 & 2 & 5.714 \\
\hline Y. Yu & Shanghai Jiao Tong University, China & 35 & 216 & 6.17 & 8 & 11 & 31.43 \\
\hline C.C. Yang & Chinese University of Hong Kong & 35 & 72 & 2.06 & 4 & 4 & 11.43 \\
\hline J. Caverlee & Texas A \& M University, USA & 35 & 287 & 8.2 & 7 & 1 & 2.857 \\
\hline H. Sundaram & Arizona State University, USA & 34 & 452 & 13.3 & 10 & 4 & 11.76 \\
\hline Total of 25 authors & & 1084 & 14,996 & 13.8 & 9.92 & 318 & 29.34 \\
\hline Total of the world & & 46,354 & & & & & \\
\hline Share of 25 authors in world output & & 2.34 & & & & & \\
\hline
\end{tabular}

$\mathrm{TP}=$ Total papers, $\mathrm{TC}=$ Total citations, $\mathrm{ACPP}=$ Average citation per paper, $\mathrm{HI}=\mathrm{H}$-index, $\mathrm{ICP}=$ International collaborative papers 
Table 9: Top 30 most productive journals contributing to social media research during 2004-2013

\begin{tabular}{|c|c|}
\hline Name of the journal & Count of papers \\
\hline Computers in Human Behavior & 375 \\
\hline First Monday & 215 \\
\hline Journal of Medical Internet Research & 192 \\
\hline Cyberpsychology Behavior, and Social Networking & 172 \\
\hline PLOS One & 165 \\
\hline Information, Communication, and Society & 153 \\
\hline Public Relations Review & 142 \\
\hline New Media and Society & 138 \\
\hline Proceedings of the ASIST Annual Meeting & 135 \\
\hline Journal of Computer Mediated Communication & 113 \\
\hline $\begin{array}{l}\text { Journal of American Society for Information } \\
\text { Science and Technology }\end{array}$ & 100 \\
\hline Multimedia Tools and Applications & 90 \\
\hline Expert Systems and Applications & 90 \\
\hline IEEE Transactions on Multimedia & 67 \\
\hline Communications of the ACM & 65 \\
\hline Computers and Education & 64 \\
\hline Decision Support Systems & 64 \\
\hline Nature & 62 \\
\hline International Journal of Web Based Communities & 59 \\
\hline Social Science Computers Review & 57 \\
\hline Online Information Review & 56 \\
\hline Lancet & 55 \\
\hline Professional De La Informacion & 53 \\
\hline American Behavioral Scientist & 53 \\
\hline Journal of Computational Information Systems & 53 \\
\hline Journal of Information Technology and Politics & 52 \\
\hline British Journal of Educational Technology & 52 \\
\hline Worldwide Web & 52 \\
\hline IEEE Intelligent Systems & 50 \\
\hline Australasian Journal of Educational Technology & 47 \\
\hline Total of 30 Journals & 2994 \\
\hline Total publications in journals of the world & 21,015 \\
\hline
\end{tabular}

16 papers appeared in the Journal of Computer Mediated Communications, followed by Computers in Human Behavior (12), New Media and Society (7), Journal of American Society for Information Science and Technology (6), Cyberpsychology and Behavior, Communications of the ACM and Communication and Education (5 each), Web Semantics, Pediatrics, First Monday, Business Horizon, Science, Journal of Applied Development Psychology and International Journal of Human-Computer Studies (3 each), Information, Communication and Society, Learning, Media and Technology, IEEE Transaction on Multimedia, Nature, Journal of Medical Internet Research, PLOS One, Journal of Service Research, Journal of Marketing, Tourism Management and Journal of General Internal Medicine (2 each). The remaining 68 journals published 1 paper each. Around 439 institutions participated in these highly cited papers. The publication productivity averaged to 1.65 paper per institution (with 152 papers by 1 organization, 78 papers by 2 organizations, 24 papers by 3 organizations, 6 papers by 4 organizations, 2 papers each by 5 organizations, and 1 paper each by 40 organizations). Among the significant organizations, the largest number of papers (11 each) were from Stanford University, Cornell University, and Yahoo Research Labs from USA, followed by University of Texas at Austin, USA (8 papers), Carnegie Mellon University, USA (7 papers), University of Maryland, USA and University of California, Berkley (6 papers each), University of Illinois, USA and Northwestern University, USA (4 papers each), University of Toronto, Canada, University of Amsterdam, The Netherlands, Columbia University in the City of New York, IBM Research, USA, University of California, Irvine, USA and University of Southern California, USA (3 papers each), etc.

A total of 816 authors participated in publishing highly cited papers, averaging 3.07 authors per paper. Seventy-six papers were contributed by 2 authors each, 69 papers by 3 authors each, 48 papers by 1 author each, 36 papers by 4 authors each, 16 papers by 5 authors each, 9 papers by 7 authors each, 5 papers by 6 authors each, 3 papers by 9 authors each, 2 papers by 11 authors each, and 1 paper each by 15 and 28 authors). Among the top authors contributing the largest number of papers (8 each) were D. Boyd and J. Leskovec, followed by N. B. Elison and J. Kleinberg (6 papers each), M. Thelwall, C. Lampe, and M. Naaman (5 papers each), M. Cha, L. Backstrom (3 papers each), X. S. Hua, C. Castillo, Zhao (2 papers each), etc., A list of top 10 highly cited papers are depicted in Table 10.

\section{SUMMARY}

The total world output on social media research covering the period 2001-2014 cumulated to 46354 publications, logging average annual $14.61 \%$ growth. A total of 142 countries participated in social media research during 2001-2014. The distribution publications output is skewed; the top 15 most productive countries accounted for the bulk share $82.40 \%$ to the world output. The USA is the world leader in social media research, accounting for the largest share $(32.40 \%)$. The remaining top 14 countries are China (8.09\%); the United Kingdom (7.67\%); Germany (4.94\%); Australia (4.30\%); Canada, Spain, and Japan (from $3.04 \%$ to $3.55 \%$ ); Italy, Taiwan, France, India, The Netherlands, and South Korea (from $2.06 \%$ to $2.58 \%$ ); and Singapore $(1.70 \%)$. The USA is also the world leader for its quality output in this area registering the largest citation impact ( 7.44 citations per paper), followed by the 
Table 10: Top 10 highly cited papers on social media research, 2001-2014

\begin{tabular}{|c|c|c|c|}
\hline Name of authors & Title of the paper & Source of publications & Number of citations \\
\hline Boyd, D.M., Ellison, N.B & $\begin{array}{l}\text { Social network sites: Definition, history, } \\
\text { and scholarship (article) }\end{array}$ & $\begin{array}{l}\text { Journal of Computer-Mediated } \\
\text { Communication, 2007, } 13(1), 210-230\end{array}$ & 2780 \\
\hline $\begin{array}{l}\text { Ellison, N.B., Steinfield, } \\
\text { C., Lampe, C }\end{array}$ & $\begin{array}{l}\text { The benefits of facebook "friends:" } \\
\text { Social capital and college students use } \\
\text { of online social network sites (article) }\end{array}$ & $\begin{array}{l}\text { Journal of Computer-Mediated } \\
\text { Communication, 2007, } 12 \text { (4), pp. 1143-1168 }\end{array}$ & 1729 \\
\hline Pang, B., Lee, L. & $\begin{array}{l}\text { Opinion mining and sentiment } \\
\text { analysis (article) }\end{array}$ & $\begin{array}{l}\text { Foundations and Trends in Information } \\
\text { Retrieval, 2008, } 2 \text { (1-2), pp. 1-135 }\end{array}$ & 1371 \\
\hline $\begin{array}{l}\text { Kaplan, A.M., } \\
\text { Haenlein, M }\end{array}$ & $\begin{array}{l}\text { Users of the world, unite! The } \\
\text { challenges and opportunities of Social } \\
\text { Media (article) }\end{array}$ & Business Horizons, 2010, 53 (1), pp. 59-68 & 1194 \\
\hline $\begin{array}{l}\text { Kwak, H., Lee, C., } \\
\text { Park, H., Moon, S. }\end{array}$ & $\begin{array}{l}\text { What is Twitter, a social network or a } \\
\text { news media? (conference paper) }\end{array}$ & $\begin{array}{l}\text { Proceedings of the } 19^{\text {th }} \text { International } \\
\text { Conference on World Wide Web, } \\
\text { WWW' } 10,2010 \text {, pp. 591-600 }\end{array}$ & 850 \\
\hline Wales, J. & $\begin{array}{l}\text { Internet encyclopedias go head to } \\
\text { head (short survey) }\end{array}$ & Nature, 2005, 438 (7070), 900-901 & 648 \\
\hline $\begin{array}{l}\text { Bizer, C., Lehmann, J., } \\
\text { Kobilarov, G., et al. }\end{array}$ & $\begin{array}{l}\text { DBpedia - A crystallization point for the } \\
\text { Web of Data (article) }\end{array}$ & $\begin{array}{l}\text { Journal of Web Semantics, 2009, } 7 \text { (3), } \\
\text { pp. 154-165 }\end{array}$ & 571 \\
\hline $\begin{array}{l}\text { Java, A., Song, X., } \\
\text { Finin, T., Tseng, B. }\end{array}$ & $\begin{array}{l}\text { Why we Twitter: Understanding } \\
\text { microblogging usage and } \\
\text { communities (conference paper) }\end{array}$ & $\begin{array}{l}\text { Joint Ninth WebKDD and First SNA-KDD } 2007 \\
\text { Workshop on Web Mining and Social Network } \\
\text { Analysis, pp. 56-65 }\end{array}$ & 529 \\
\hline $\begin{array}{l}\text { Sakaki, T., Okazaki, M., } \\
\text { Matsuo, Y. }\end{array}$ & $\begin{array}{l}\text { Earthquake shakes Twitter users: } \\
\text { Real-time event detection by social } \\
\text { sensors (Conference Paper) }\end{array}$ & $\begin{array}{l}\text { Proceedings of the } 19^{\text {th }} \text { International Conference } \\
\text { on World Wide Web, WWW ' } 10 \text {, pp. 851-860 }\end{array}$ & 522 \\
\hline $\begin{array}{l}\text { Mislove, A., Marcon, M., } \\
\text { Gummadi, K.P., et al. }\end{array}$ & $\begin{array}{l}\text { Measurement and analysis of online } \\
\text { social networks (conference paper) }\end{array}$ & $\begin{array}{l}\text { Proceedings of the ACM SIGCOMM Internet } \\
\text { Measurement Conference, IMC, 2007, pp. 29-42 }\end{array}$ & 479 \\
\hline
\end{tabular}

United Kingdom (5.66), The Netherlands (5.59), Canada (5.46), Germany (5.31), France (5.16), etc. Computer science, among various broad subjects, accounted for the largest publications share $(55.22 \%)$, followed by social sciences $(26.55 \%)$, engineering $(13.52 \%)$, medicine (10.14\%), business, management and accounting $(8.72 \%)$, arts and humanities (5.95\%), psychology (3.68\%), etc. The septennial research activity as measured on activity index indicator went up in computer science, social sciences, medicine, arts and humanities, psychology and decision sciences, but it went down in engineering, business, management and accounting, economics, econometrics, and finance during septennial periods from 2001-2007 to 2008-2014. Blogs contributed the largest publication share $(27.45 \%)$, followed by Facebook (16.75\%), Twitter (15.86\%), Wikipedia (10.58\%), YouTube (7.24\%), Flickr (3.94\%), MySpace (1.73\%), LinkedIn (1.21\%), etc. Online social network was seen as the most significant search term; it accounted for the largest number of hits (10395) followed by social media (9605), social networks (6076), internet (6352), Facebook (3495), Wikipedia (2973), blogs (2535), world wide web (2524), Twitter (2041), data mining (1997), information retrieval (1956), websites (1922), Web 2.0 (1684), information system (1520), YouTube (1279), blogging (1208), etc., during 2001-2014.

The top 25 most productive organizations together contributed $12.46 \%$ share, registered average productivity of 231.01 per organization, average citation impact 9.24 citations per paper, average h-index of 21 score, and an average of share $26.14 \%$ international collaborative publications. The top 25 most productive authors contributed $2.34 \%$ share, registered an average productivity of 43.06 per author, average citation impact of 13.8 citations per paper, average h-index of 9.92 score, and average share of $29.34 \%$ international collaborative publications during 2001-2014. The top 30 most productive journals together contributed $14.25 \%$ share. Of the 46354 total world publications in social media research, only 266 papers $(0.57 \%)$ were cited 100 or more times each since publication till February 2015. Together these highly cited papers accounted for 57462 citations, averaging 216.02 citations per paper. Of the total 266 highly cited papers, 140 were single-institution papers (zero collaboratives), 89 national collaborative papers, and 37 international collaborative papers. United States accounted for the highest number (173) of highly cited papers, followed by the U.K. (23), Germany (21), Canada (14), The Netherlands (9), Spain (7), Australia and France (5 each), and South Korea, Hong Kong, Switzerland, and Israel (4 each). Of the 266 highly cited papers, 146 appeared as articles, 100 as conference papers, 14 as reviews, 4 as books, 1 as editorial, and 1 as short survey. The 162 high cited papers have appeared in 84 journals, of which 16 appeared in the Journal of Computer Mediated Communications, followed by Computers in Human Behavior (12), New Media and Society (7), Journal 
of the American Society for Information Science and Technology (6), etc.

\section{CONCLUSION}

The web first empowered the public with vast amounts of information, but social media gave something, even more powerful: Personal and professional connectivity as a way to connect friends and family online. Organizations quickly saw in it game-changing advantage for marketing, advertising, public care, and feedback. The social web can certainly help governments to run national programs much more effectively. Using this media, they can communicate within their own walls and also communicate with the public. They have a platform where they can get feedback and opinion, and also get into discussion and collaboration with the public on policies, programs, and schemes. For professional communities, social media can provide contents to keep up to date with current research, popular sciences covering broader issues of science policy, funding, publishing, and personal career development. Certain social media tools have become invaluable for professional networking either within subject fields or across different disciplines and professions. Actively participating in social media networks allows scientists to disseminate research findings quickly and effectively as well as raise their own profile, or of their research groups or of their parent institutions. More importantly, the interactive nature of the medium can be highly beneficial for scientists by offering them new perspectives on their own research through dialog with peers and nonpeers, and helping them to establish new collaborations.

In view of the benefits of social media to research, governments, and citizens, it is suggested that governments should consider using social media as a part of overall policy and communication mix in providing and promoting good governance, open up public access to government officials and policy makers, and create new ways of government working in partnership with public. Moreover, it is the time that researchers come up with a set of new indicators to effectively gauge the impact of new technologies and media on questions of social, economic, and political change.

\section{Financial Support and Sponsorship}

Nil.

\section{Conflicts of Interest}

There are no conflicts of interest.

\section{REFERENCES}

1. Simon K. Digital, Social and Mobile Worldwide in 2015; 21 January, 2015. Available from: http://www.wearesocial.net/blog/2015/01/ digital-social-mobile-worldwide-2015/. [Last accessed on 2015 Mar 10].

2. Kaplan AM, Haenlein M. Users of the world, unite! The challenges and opportunities of social media. Bus Horiz 2010;53:59-68.

3. Drew H. Complete History of Social Media: Then and Now. Available from: http://www.smallbiztrends.com/2013/05/ the-complete-history-of-social-media-infographic.html. [Last accessed on 2015 Dec 15].

4. Bell D. The Social Media Handbook. Tebbo: Emereo Publishing; 2012. p. 514.

5. Coursaris CK, Van Osch W. A scientometric analysis of social media research (2004-2011). Scientometrics 2014;101:357-89.

6. Gan, Chunmei. A Bibliometric Analysis of Social Media Research from the Perspective of Library and Information Science. In. Li, Hongxiu, Mäntymäki, Matti, Zhang, Xianfeng, eds. Digital Services and Information Intelligence. 13 $3^{\text {th }}$ IFIP WG 6.11 Conference on e-Business, e-Services, and e-Society, I3E 2014, Sanya, China, November 28-30, 2014, Proceedings. Springer, 2014.23-32. 